\title{
cAMP Response Element-Binding Protein in the Amygdala Is Required for Long- but not Short-Term Conditioned Taste Aversion Memory
}

\author{
Raphael Lamprecht, Shoshi Hazvi, and Yadin Dudai \\ Department of Neurobiology, The Weizmann Institute of Science, Rehovot 76100, Israel
}

In conditioned taste aversion (CTA) organisms learn to avoid a taste if the first encounter with that taste is followed by transient poisoning. The neural mechanisms that subserve this robust and long-lasting association of taste and malaise have not yet been elucidated, but several brain areas have been implicated in the process, including the amygdala. In this study we investigated the role of amygdala in general, and the CAMP response element-binding protein (CREB) in the amygdala in particular, in CTA learning and memory. Toward that end, we combined antisense technology in vivo with behavioral, molecular, and histochemical analysis. Local microinjection of phosphorothioate-modified oligodeoxynucleotides (ODNs) antisense to CREB into the rat amygdala several hours before CTA training transiently reduced the level of CREB protein during training and impaired CTA memory when tested 3-5 d later. In comparison, sense ODNs had no effect on memory. The effect of antisense was not attributable to differential tissue damage and was sitespecific. CREB antisense in the amygdala had no effect on retrieval of CTA memory once it had been formed, and did not affect short-term CTA memory. We propose that the amygdala, specifically the central nucleus, is required for the establishment of long-term CTA memory in the behaving rat; that the process involves long-term changes, subserved by CRE-regulated gene expression, in amygdala neurons; and that the amygdala may retain some CTA-relevant information over time rather than merely modulating the gustatory trace during acquisition of CTA.

Key words: conditioned taste aversion; amygdala; CREB; learning; short-term memory; long-term memory
In conditioned taste aversion (CTA), organisms learn to avoid a taste if the first encounter with that taste is followed by malaise (Garcia et al., 1955; Bures et al., 1988). CTA presents to memory research remarkable opportunities but also a unique challenge. The acquisition of CTA is fast (a single trial), and its memory is robust (high signal-to-noise ratio in the behavioral response) and long-lasting (up to a lifetime). These properties facilitate correlation of molecular and cellular events in brain with phases of learning, consolidation, retention, and retrieval in a natural behavioral situation. Moreover, conditioning in CTA tolerates a delay of hours between the taste [conditioned stimulus (CS)] and the malaise [unconditioned stimulus (UCS)]. This permits separation in time of the mechanisms of acquisition of information about the taste from the association of that information with a reinforcer. Such separation provides a useful system for investigating incidental learning, i.e., learning in the absence of an exogenous reinforcer (Hebb, 1949), a very common but rather neglected type of learning. However, the uniquely long CS-UCS interval in CTA, so different from classical instances of conditioning that demand tight temporal association (less than seconds), also raises the issue of how it is that the memory of a taste remains specifically associable with malaise for many hours. Not surprisingly, CTA, although well known to farmers, encountered

\footnotetext{
Received March 25, 1997; revised Aug. 12, 1997; accepted Aug. 20, 1997.

We thank Z. Kam for advice on the use of the digital microscope system, The Carl Dominic Center for Brain Research, Rehovot, and the US-Israel Binational Science Foundation (BSF), Jerusalem, for support, and J. LeDoux and M. Segal for comments on this manuscript.

Correspondence should be addressed to Dr. Yadin Dudai at the above address.

Copyright (C) 1997 Society for Neuroscience $0270-6474 / 97 / 178443-08 \$ 05.00 / 0$
}

difficulties in being accepted as a paradigm of conditioning in experimental psychology (Garcia, 1981).

Whereas the behavioral characteristics of CTA have been extensively investigated (Domjan, 1980; Bures et al., 1988), much less is known about brain systems that subserve it and the molecular and cellular mechanisms that embody CTA in these areas. It is generally accepted that the gustatory cortex plays a role in the processing and memory of the taste stimulus, the parabrachial nucleus in the association between the taste and malaise, and the amygdala in the integration and expression of CTA behavior (for review, see Yamamoto et al., 1994). The role of amygdala is especially interesting, because this brain structure has been implicated in other types of learning, especially aversive and emotional conditioning (Davis, 1992; LeDoux, 1993; McGaugh et al., 1993). We recently reported that transient inhibition of protein synthesis in the central amygdala during CTA training blocks CTA memory, and that local microinjection into the amygdala of antisense oligodeoxynucleotides (ODNs) to the immediate-early gene (IEG) c-fos has a similar effect (Lamprecht and Dudai, 1996). We have now combined the antisense technology in vivo (for review, see Cirelli et al., 1995) with behavioral, molecular, and histochemical analysis to show that in the amygdala, the cAMP response element-binding protein (CREB), implicated in multiple types of neuronal plasticity (Frank and Greenberg, 1994; Carew, 1996), is essential for long-term CTA memory, but not for short-term memory or for the retrieval of long-term memory once it has been formed. Our findings suggest that CTA shares mechanisms with other, more "conventional" forms of learning, and that long-term changes involving CRE-regulated gene expression take place in the amygdala in encoding CTA memory. 


\section{MATERIALS AND METHODS}

\section{Animals}

Wistar rats (2-month-old males, 200-250 gm) were caged individually at $22^{\circ} \mathrm{C}$ under $12 \mathrm{hr}$ light/dark cycles. Water and food were available ad libitum unless otherwise indicated.

\section{Reagents}

The phosphorothioate-modified ODNs were synthesized at the Biological Services of the Weizmann Institute of Science. The sequence for CREB antisense was 5'-TGGTCATCTAGTCACCGGTG-3', and that for CREB sense was 5'-CACCGGTGACTAGATGACCA-3'. Fluorescein isothiocyanate-labeled CREB antisense (FITC-CREB) was from Biosource (Camarillo, CA). ODNs were microinjected in physiological saline $(0.9 \% \mathrm{NaCl})$. Anti-CREB antibody (06-504) was from Upstate Biotechnology (Lake Placid, NY). Anti-activation transcription factor 2 (ATF-2) antibody (SC-242) and anti-protein kinase $\mathrm{C}_{\gamma}\left(\mathrm{PKC}_{\gamma}\right)$ antibody (SC-211) were from Santa Cruz Biotechnology (Santa Cruz, CA). Biotinylated goat anti-rabbit antibody (BA-1000) and avidin DH-biotinylated horseradish peroxidase (HRP) H reagents (Vectastain, PK-6100) were from Vector Laboratories (Burlingame, CA). HRP-protein A (NA-9120) and an enhanced chemoluminiscence (ECL) kit (RPN 2106) were from Amersham (Buckinghamshire, UK). Anti- $\alpha$-tubulin antibody (T-9026), HRP-goat anti-mouse antibody (A-9917), cytochrome $c$ (C-7752), 3',3' diaminobenzidine tetrahydrochloride (DAB; Sigma Fast tablets, D4293), and catalase (C-40) were from Sigma (St. Louis, MO). All other chemicals were of analytical grade or the highest grade available.

\section{Behavioral procedures}

In CTA, saccharin $(0.1 \% \mathrm{w} / \mathrm{v}$, sodium salt) was used as an unfamiliar taste unless otherwise indicated, and intraperitoneal $\mathrm{LiCl}(0.15 \mathrm{M}, 2 \%$ body weight) was used as a malaise-inducing agent. The CTA protocol was essentially as described by Rosenblum et al. (1993). In brief, rats were trained over $4 \mathrm{~d}$ to get their daily water ration within $10 \mathrm{~min} / \mathrm{d}$ from 2 pipettes, each containing $10 \mathrm{ml}$. On day 5 (conditioning day), the rats were presented with saccharin instead of water. Forty minutes later, they were injected with $\mathrm{LiCl}$ intraperitoneally. On days 6-7 or 6-8 (rest days), the rats were presented daily for $10 \mathrm{~min}$ with two pipettes containing $10 \mathrm{ml}$ of water each. In the test, performed in an extinction mode on three successive days (days 8-10 or 9-11), the rats were presented daily with an array of six pipettes, three containing $4 \mathrm{ml}$ of saccharin and three containing $4 \mathrm{ml}$ water, and their liquid consumption was recorded. In the experiments designed to test both short- and long-term memory, all rats were trained as above, except that on day 5 (conditioning day) they were presented with $5 \mathrm{ml}$ of saccharin, and 2 or $4 \mathrm{hr}$ after the injection of $\mathrm{LiCl}$ intraperitoneally they were presented with an array of six pipettes, three containing $3 \mathrm{ml}$ of saccharin and three containing $3 \mathrm{ml}$ of water for $10 \mathrm{~min}$, and their liquid consumption was recorded. The same rats were again presented $72 \mathrm{hr}$ after the injection of $\mathrm{LiCl}$ with an array of six pipettes, three containing $4 \mathrm{ml}$ of saccharin and three containing $4 \mathrm{ml}$ water for $15 \mathrm{~min}$, and their liquid consumption was recorded again. The aversion index (Rosenblum et al., 1993) was defined as $\{$ [milliliters of water/(milliliters of water + milliliters of saccharin) $] \times$ $100\}$ consumed in the test; that is, 50 is chance level, and the higher the aversive index, the more the rats prefer water to saccharin.

To follow the time course of malaise induced by $\mathrm{LiCl}$ injection in CTA training, rats were injected with $\mathrm{LiCl}$ intraperitoneally as above or with saline as control, placed individually in transparent cages, and observed. Two behavioral responses were monitored at intervals of $30 \mathrm{~min}$, up to 330 min after the injection: lying on the belly (LOB), characterized by little or no movement and a prone, flaccid posture with the chin on the floor of the cage (Meachum and Bernstein, 1990); and rearing, expressed as lifting the forepaws simultaneously off the floor in the absence of grooming (Parker, 1982).

\section{Surgical procedures}

Microinjection into the amygdala was performed via chronically implanted cannulas. For surgery, rats were anesthetized with Equithesin $(5.6 \mathrm{ml} / \mathrm{kg})$, restrained in a stereotaxic apparatus (Kopf), and implanted bilaterally with guide cannulas (stainless steel, 23 gauge) aimed at the central amygdaloid nucleus [ $\mathrm{CeA}$; coordinates in reference to bregma: anteroposterior (AP), -2.3 ; lateral (L) \pm 4 ; and dorsoventral (DV), -7.5] (Paxinos and Watson, 1986). In a set of control experiments, the guide cannulas were implanted bilaterally in the basal ganglia (coordinates in reference to bregma: AP, -2.3 ; L, \pm 4 ; and DV, -5.5 ). The cannulae were fixed in place with acrylic dental cement and secured by two skull screws. A stylus was placed in the guide cannula to prevent clogging. Animals were allowed 1 week to recuperate before being subjected to experimental manipulations.

\section{Microinjection}

Microinjection of ODNs (4 $\mathrm{nmol}$ in $2 \mu \mathrm{l}$ /hemisphere) was performed at the times indicated in Results. The stylus was removed from the guide cannula, and a 28 gauge injection cannula was carefully inserted and lowered $1 \mathrm{~mm}$ below the tip of the guide cannula. We chose these injection coordinates, which are just at the bottom of the $\mathrm{CeA}$, because in our experience this on the one hand minimized the injection lesion in the $\mathrm{CeA}$ and on the other maximized the diffusion of the injected solution into the $\mathrm{CeA}$, since the solution tended to diff use dorsolaterally to the tip of the injection cannula (see below). The injection cannula was connected via PE20 tubing to a Hamilton microsyringe driven by a microinjection pump (Carnegie Medicine CMA 100) at a rate of 0.5 $\mu \mathrm{l} / \mathrm{min}$. After microinjection, the injection cannula was left for an additional 1 min before withdrawal to reduce efflux of injection liquid along the injection tract. No tremors or seizures were detected during or after the injection.

In a preliminary set of experiments, using india ink, we estimated the sphere of diffusion of the solution microinjected into the amygdala as $1.5 \pm 0.4 \mathrm{~mm}^{3}(n=4)$. The solution diffused in and around the CeA. Occasionally, it also extended into one or more of the following structures: the ventral horn of the caudate putamen, the nucleus basalis of Meynert, the dorsal portion of the internal capsule, the intercalated amygdaloid nuclei, and the basolateral amygdala. The only common feature of the microinjections, however, was the bilateral involvement of substantial portions of the CeA (also see Lamprecht and Dudai, 1996). Microinjection of FITC-CREB antisense ODNs unveiled a more focused high-concentration sphere of $0.72 \pm 0.04 \mathrm{~mm}^{3}(n=3)$, spreading mainly dorsolaterally to the tip of the cannula and uncovering mostly the $\mathrm{CeA}$ (both medial and lateral subnuclei), but also penetrating the intercalated nuclei and limited portions of the basomedial and basolateral amygdala (Fig. 1A).

\section{Histochemistry and image analysis}

Images of FITC-CREB antisense were recorded with a Zeiss Axioskop microscope coupled to a cooled CCD camera (Photometrics, Tuscon, AZ). The computerized microscope system was as described by Kam et al. (1993). The FITC filters used were from Omega (Brattleboro, VT). Digital images were acquired and stored using the Priism software (Applied Precision, Issaqua, WA) (Kam et al., 1992). Composite images were taken over overlapping regions and fitted using Photoshop 3.0.4 (Adobe, Mountain View, CA).

For immunohistochemical localization of CREB, rats were given Equithesin $(5.6 \mathrm{ml} / \mathrm{kg}$, i.p.) $14 \mathrm{hr}$ after the microinjection of the ODNs and perfused intracardially by gravity drip infusion with PBS followed by cold $\left(4^{\circ} \mathrm{C}\right)$, fresh $2.5 \%$ paraformaldehyde and $5 \%$ sucrose in PBS, $\mathrm{pH} 7.4$. The brain was post-fixed for $48 \mathrm{hr}$ in $1 \%$ paraformaldehyde and $30 \%$ sucrose in PBS at $4^{\circ} \mathrm{C}$. Post-fixed brains were sectioned coronally on a freezing microtome at a thickness of $50 \mu \mathrm{m}$. Sections were washed three times (5 min each) with PBS and immersed for $30 \mathrm{~min}$ in $49.5 \%$ methanol, $49.5 \%$ PBS, and $0.9 \% \mathrm{H}_{2} \mathrm{O}_{2}$, followed by washing five times (5 min each) with PBS and immersion for $20 \mathrm{~min}$ in $0.15 \mathrm{M}$ glycine in PBS, $\mathrm{pH}$ 7.4. The sections were then blocked with $20 \%$ normal goat serum (NGS) in PBS and $0.5 \%$ Triton X-100 for $1-3 \mathrm{hr}$ at $37^{\circ} \mathrm{C}$, followed by 16 $\mathrm{hr}$ incubation at room temperature with the anti-CREB polyclonal antibody (1:500) and 2\% NGS in PBS. The sections were washed (three times, 5 min each) with PBS and incubated with biotinylated goat anti-rabbit antibody (1:200) and 2\% NGS in PBS for $1.5 \mathrm{hr}$ at room temperature, followed by three additional PBS washings $(5 \mathrm{~min}$ each). Finally, an avidin DH-biotinylated horseradish peroxidase $\mathrm{H}$ complex (1:50) was added for $1.5 \mathrm{hr}$. The sections were then washed once with PBS and twice with $50 \mathrm{~mm}$ Tris-Cl buffer, $\mathrm{pH}$ 7.5. Peroxidase activity was determined by reaction with a mixture containing $0.7 \mathrm{mg} / \mathrm{ml}$ DAB and 2 $\mathrm{mg} / \mathrm{ml}$ urea- $\mathrm{H}_{2} \mathrm{O}_{2}$ in $60 \mathrm{~mm}$ Tris-Cl. The sections were washed three times with Tris buffer, mounted on slides, dehydrated by successive rinses of 70, 95, and 100 ethanol (two times) and 100\% xylene (two times), and covered with Permount.

For cytochrome oxidase staining, rats were treated with Equithesin as above $4 \mathrm{~d}$ after the microinjection of ODNs into the amygdala and perfused intracardially by gravity drip infusion with PBS followed by cold $2.5 \%$ glutaraldehyde, $0.5 \%$ paraformaldehyde, and $5 \%$ sucrose in 


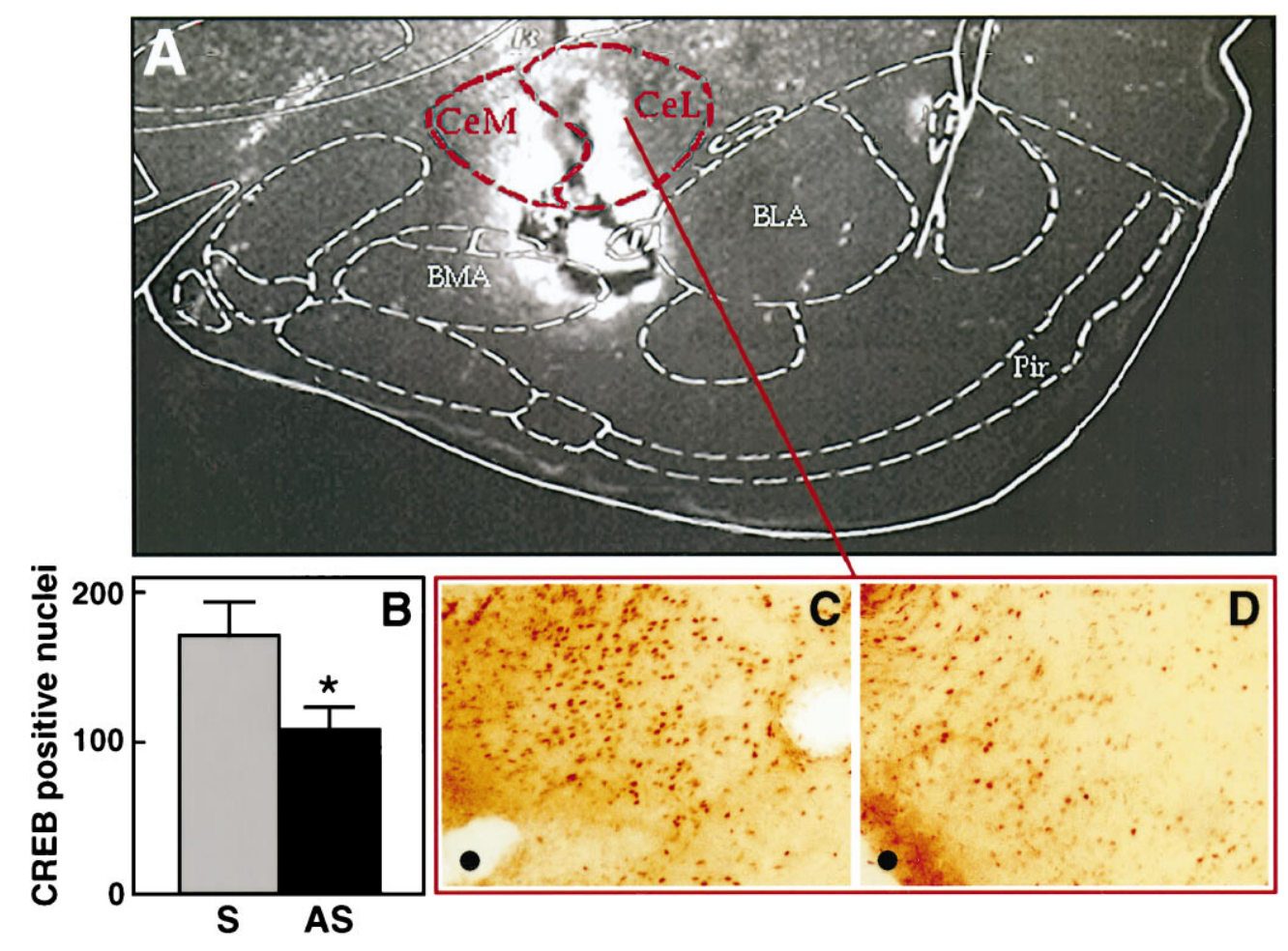

Figure 1. Sphere of diffusion of microinjected CREB antisense ODNs into the amygdala $(A)$ and reduction in CREBpositive nuclei by CREB antisense $(B-$ $D)$. $A$, FITC-labeled CREB antisense was microinjected, and the fluorescence image recorded as detailed in Materials and Methods. CeM, Central amygdaloid nucleus, medial division; $C e L$, central amygdaloid nucleus, lateral division; $B M A$, basomedial amygdaloid nucleus, anterior part; $B L A$, basolateral amygdaloid nucleus, anterior part; Pir, piriform cortex. The microinjection cannula track and the lesion induced by the tip of the cannula are seen within the sphere of FITC diffusion. The schematic anatomical map is adapted from that of Paxinos and Watson (1986). B, The number of CREB-positive nuclei in the amygdala of CREB antisense $(A S)$ versus sense $(S)$ microinjected rats. In both groups CREB antisense was microinjected into the amygdala $14 \mathrm{hr}$ before CTA training; $n=$ 4 in each group. The asterisk indicates significance for pair comparison in which a Scheffe contrast test was used with an $\alpha$ of 0.05 . $C, D$, Immunohistochemistry of CREB using CREB antibodies in CREB sense $(C)$ and antisense $(D)$ microinjected rats. The selected fields were taken from the region of the $\mathrm{CeL}$, indicated in $A$; dot, edge of the microinjection cannula-induced lesion.
PBS. Brains were post-fixed for $12 \mathrm{hr}$ in $2.5 \%$ glutaraldehyde, $0.5 \%$ paraformaldehyde, and $15 \%$ sucrose in PBS at $4^{\circ} \mathrm{C}$. Post-fixed brains were sectioned coronally on a freezing microtome at $50 \mu \mathrm{m}$ thickness, washed four times, $5 \mathrm{~min}$ each, in $0.1 \mathrm{M}$ phosphate buffer, $\mathrm{pH}$ 7.6, and incubated for $3-24 \mathrm{hr}$ at $40^{\circ} \mathrm{C}$ in an oxygenated reaction mixture containing $0.7 \mathrm{mg} / \mathrm{ml} \mathrm{DAB}, 75 \mu \mathrm{g} / \mathrm{ml}$ cytochrome $c$, and $2000 \mathrm{U} / \mathrm{ml}$ catalase (in $0.1 \%$ thymol) in $0.1 \mathrm{~m}$ phosphate buffer, $\mathrm{pH}$ 7.6. The sections were then washed four times in $0.1 \mathrm{~m}$ phosphate buffer, $\mathrm{pH} 7.6$, mounted on slides, dehydrated by successive rinses in $50,70,95$, and $100 \%$ ethanol (two times) and $100 \%$ xylene (three times), and covered with Permount.

For Nissl staining, brains were processed as above. Sections were mounted on slides and dehydrated by successive rinses in 95 (three times) and $100 \%$ (three times) ethanol, $2 \mathrm{~min}$ each, followed by $30 \mathrm{~min}$ in chloroform. The slides were then rehydrated by successive rinses in 100 (three times), 95 (three times), and $70 \%$ ethanol ( 2 min each). The slides were stained with $0.25 \%$ cresyl violet in $25 \%$ ethanol, $231 \mathrm{~mm}$ acetic acid, and $18.7 \mathrm{~mm}$ sodium acetate for $2 \mathrm{~min}$, washed in water, rinsed $1 \mathrm{~min}$ in $25 \%$ ethanol, bleached with $50 \%$ ethanol and $0.5 \%$ acetic acid, and then rinsed in 70, 95 (once), and 100\% (three times) ethanol for $2 \mathrm{~min}$ and $100 \%$ xylene (three times) for $15 \mathrm{~min}$, and covered with Permount. A Nikon Labophot microscope and Image 1.41 software (National Institutes of Health, Bethesda, MD) were used for histochemical analysis.

\section{Immunoblotting}

CREB antisense ODNs were microinjected as above into the amygdala of one hemisphere, and CREB sense ODNs were injected into the amygdala of the contralateral hemisphere. At 14 or $24 \mathrm{hr}$ after injection, the rats were given Equithesin $(5.6 \mathrm{ml} / \mathrm{kg}$, i.p.) and perfused intracardially with PBS for $3 \mathrm{~min}$. The brain was rapidly removed, frozen on dry ice, and sectioned in a cryostat until the tip of the microinjection cannula was visualized. Tissue from the tip of the cannula at each hemisphere was punched out with a 1-mm-length 15-gauge stainless steel syringe needle blunted at the tip (inner diameter, $1.3 \mathrm{~mm}$ ). The tissue thus obtained was homogenized in a glass-Teflon homogenizer in SDS sample buffer containing $10 \%$ glycerol, $5 \% \beta$-mercaptoethanol, and $2.3 \%$ SDS in $62.5 \mathrm{~mm}$ Tris-Cl, $\mathrm{pH}$ 6.8. Aliquots containing equal amounts of protein $(25-50$ $\mu \mathrm{g})$ were subjected to SDS polyacrylamide gel electrophoresis in $12 \%$ polyacrylamide (Laemmli, 1970) and Western blotting (Burnette, 1981). After protein transfer, the nitrocellulose was treated as described below either in Protocol 1 (anti-CREB or anti- $\alpha$-tubulin antibodies) or in Protocol 2 (anti-ATF-2 or anti-PKC ${ }_{\gamma}$ antibodies).

Protocol 1. The blot was blocked with $1 \%$ BSA in washing buffer $(0.9 \%$ $\mathrm{NaCl}, 0.05 \%$ Tween 20 , and $10 \mathrm{~mm}$ Tris, $\mathrm{pH} \mathrm{7.6)} \mathrm{for} 1 \mathrm{hr}$ at room temperature and reacted overnight at $4^{\circ} \mathrm{C}$ or for $2 \mathrm{hr}$ at room temperature with anti-CREB antibody (1:1000) or anti- $\alpha$-tubulin antibody (1:5000). Blots were washed with washing buffer three times, 5 min each, followed by a $1 \mathrm{hr}$ incubation at room temperature with HRP-linked protein A $(1: 15,000)$ or HRP-linked goat anti-mouse $(1: 5000)$ for the anti-CREB antibody or the anti- $\alpha$-tubulin antibody, respectively. Blots were washed with washing buffer once for $15 \mathrm{~min}$ and then thrice for $5 \mathrm{~min}$ before being subjected to ECL analysis. The anti-CREB antibody recognized the expected $\sim 43 \mathrm{KD}$ a band (Ginty et al., 1993).

Protocol 2. The blot was blocked with $5 \%$ milk powder and $0.05 \%$ Tween 20 in TBS ( $150 \mathrm{~mm} \mathrm{NaCl}$ and $10 \mathrm{~mm}$ Tris-HCl, $\mathrm{pH} 8.0)$ for $30 \mathrm{~min}$ at room temperature, followed by a $45 \mathrm{~min}$ incubation with anti-ATF-2 (1:50) or anti-PKC $\mathrm{PK}_{\gamma}(1: 800)$ in blocking solution at room temperature. The blots were washed twice for 7 min with $0.05 \%$ Tween 20 in TBS and incubated with HRP-linked protein A $\left(\mathrm{PKC}_{\gamma}\right.$ blot) or HRP-linked goat anti-mouse (ATF-2 blot) for $30 \mathrm{~min}$ at room temperature, followed by three 5 min washes with $0.05 \%$ Tween 20 in TBS and then once with TBS. Here, too, the blots were subjected to ECL analysis.

In both protocols, quantification was performed in a Molecular Dynamics (Sunnyvale, CA) 300A densitometer. Values were calculated as percentages of the CREB, ATF-2, or $\mathrm{PKC}_{\gamma}$ proteins in the antisense-microinjected hemisphere, taking in each individual rat the value in the contralateral sense-microinjected hemisphere as $100 \%$. In each rat, the values obtained for CREB, ATF-2, or $\mathrm{PKC}_{\gamma}$ were normalized to the level of $\alpha$-tubulin, taken as a stable protein reference ( $\tau_{1 / 2} \sim$ days; Dustin, 1984).

\section{Statistics}

Differences among groups were evaluated using one-way ANOVA and for repeated measure test two-way ANOVA. For paired comparisons Scheffe contrast tests were used with an $\alpha$ level of 0.05 . 


\begin{tabular}{|c|c|c|}
\hline Protein & $14 \mathrm{hr}$ & $24 \mathrm{hr}$ \\
\hline CREB & $65 \pm 12(7)^{*}$ & $97 \pm 7(7)$ \\
\hline ATF-2 & $91 \pm 8(7)$ & $94 \pm 6(5)$ \\
\hline $\mathrm{PKC}_{\gamma}$ & $91 \pm 8(7)$ & $100 \pm 7(8)$ \\
\hline
\end{tabular}

Rats were micorinjected into the amygdala with CREB antisense into one hemisphere and sense ODNs into the contralateral hemisphere. Animals were killed 14 or $24 \mathrm{hr}$ after microinjection, amygdala tissue was excised and homogenized, and samples were subjected to immunoblotting with antibodies to CREB, ATF-2, PKC or $\alpha$-tubulin. Values are percentages of the indicated proteins in the antisensemicroinjected hemisphere, taking in each individual rat the level in the sensemicroinjected hemisphere as $100 \%$. In each rat, the values for CREB, ATF-2, and $\mathrm{PKC}_{\gamma}$ were normalized to the level of $\alpha$-tubulin. For further details see Materials and Methods. Values in parentheses are number of rats in each comparison.

* Significance of pair comparisons in which a Scheffe contrast test was used with an $\alpha$ of 0.05 .

\section{RESULTS}

\section{CREB antisense reduced the level of CREB protein in the amygdala}

We have determined the effect of CREB antisense ODNs in the amygdala on the level of the CREB protein by two methods, immunohistochemistry and immunoblotting. Immunohistochemical analysis of the central amygdala $14 \mathrm{hr}$ after the injection of antisense or sense ODNs showed that the antisense significantly reduced the level of CREB protein in the vicinity of the tip of the injection cannulas (Fig. $1 B-D$ ). The average reduction over an area of $0.5 \mathrm{~mm}^{2}$ was $37 \pm 9 \%$ (Fig. $1 B ; p<0.02$ ). Immunoblotting of tissue excised from the vicinity of the tip of the injection cannula unveiled a similar magnitude of reduction at $14 \mathrm{hr}$, which was specific to the CREB protein; the transcription factor ATF-2 and the enzyme $\mathrm{PKC}_{\gamma}$, assayed as controls, were not affected (Table 1). The level of CREB protein returned to normal within $24 \mathrm{hr}$ (Table 1).

\section{CREB antisense in the amygdala impaired CTA memory}

When microinjected into the amygdala $14 \mathrm{hr}$ before CTA training, CREB antisense markedly reduced CTA memory tested 3-5 $\mathrm{d}$ after training (Fig. 2). In contrast, CREB sense had no effect. Differences among groups were significant for all postconditioning days ( $p<0.001$ for days 1 and $2 ; p<0.002$ for day 3 of testing). The aversion index of the CREB antisense group was significantly lower than that of normal and CREB sense groups in all test days (Scheffe contrast tests). A group-by-days ANOVA demonstrated a significant difference among groups $(p<0.001)$ and a significant decrease in the aversion index over days $(p<$ $0.001)$ with no interaction effect.

\section{The effect of CREB antisense was not due to differential tissue damage}

Because ODNs may have toxic effects including tissue damage in vivo (Chiasson et al. 1994), we wanted to verify that the differential effect of CREB antisense on CTA was not attributable to differential tissue toxicity in the amygdala. Toward that end, we compared cytochrome oxidase- and Nissl-stained sections from animals microinjected into the amygdala with saline or CREB sense or CREB antisense ODNs $14 \mathrm{hr}$ before CTA training and killed $4 \mathrm{~d}$ later. The number of Nissl-stained cells and the intensity of cytochrome oxidase activity were compared in selected frames near the cannula tip and found not to differ between saline (S), CREB sense (Se), and CREB antisense (A): Nissl staining,
$544 \pm 27(\mathrm{~S}), 516 \pm 31(\mathrm{Se})$, and $525 \pm 69(\mathrm{~A})$ cells in $0.12 \mathrm{~mm}^{2}$; and cytochrome oxidase, $128 \pm 9(\mathrm{~S}), 123 \pm 4(\mathrm{Se})$, and $126 \pm 6$ (A) arbitrary pixel units $[n=3(\mathrm{~S}) ; n=4$ (Se and A)].

\section{CREB antisense did not prevent conditioning several days later}

In contrast to its effect on CTA memory when microinjected $14 \mathrm{hr}$ before training, CREB antisense had no effect on CTA memory when microinjected into the amygdala several days before training. It should be noted that ODNs are expected to degrade in vivo within 1-2 d (Chiasson et al., 1994; Konradi et al., 1994), and that in our hands the level of CREB in the CREB antisensemicroinjected amygdala indeed returned to normal within $1 \mathrm{~d}$ (Table 1).

That the time window of the effect of a single microinjection of CREB antisense on CTA training is limited became evident in two types of experiments. In experiment 1, rats were microinjected into the amygdala with CREB antisense $72 \mathrm{hr}$ before CTA training, using saccharin as the unfamiliar taste; there was no effect on CTA memory tested $3 \mathrm{~d}$ after training (aversion index of $92 \pm 5$ vs $94 \pm 1$ in CREB antisense-microinjected and control animals, respectively). In experiment 2, we wanted to reinforce the conclusions by using rats in which the blocking effect of CREB antisense on CTA had already been demonstrated once, and to generalize the findings to other tastes. We thus microinjected rats into the amygdala with CREB antisense $14 \mathrm{hr}$ before CTA training, using saccharin as the unfamiliar taste; in agreement with the data presented above, we found an impairment in CTA memory tested $3 \mathrm{~d}$ after training (aversion index of $77 \pm 6 \mathrm{vs} 95 \pm$ 1 in CREB antisense and control animals, respectively; $p<0.01$ ). However, 1 week later we reconditioned the same rats, this time using $\mathrm{NaCl}(0.1 \mathrm{M})$ as the unfamiliar taste. The aversion tested $3 \mathrm{~d}$ later was normal $(97 \pm 1)$. In a complementary experiment, using other, naive rats, we found that CREB antisense, microinjected into the amygdala $14 \mathrm{hr}$ before CTA training in which $0.1 \mathrm{M} \mathrm{NaCl}$ was used as the novel taste solution, did impair the aversion index by $(26 \pm 4) \%$ when tested $3 \mathrm{~d}$ after training, similarly to the effect on CTA to saccharin. We thus concluded that the microinjection of ODNs into the amygdala caused no residual functional damage to this structure with regard to CTA learning.

\section{The effect displayed anatomical site specificity}

Microinjection of the CREB antisense into the basal ganglia (2 $\mathrm{mm}$ above the coordinates used by us for microinjection into the amygdala), $14 \mathrm{hr}$ before CTA training, had no effect on CTA memory (Fig. 2, inset), indicating site specificity of the effect rather than general toxicity.

\section{CREB antisense affected long- but not short-term CTA memory}

Routinely, CTA memory is tested several days after training (e.g., Bermudez-Rattoni et al., 1986; Gallo et al., 1992; Rosenblum et al., 1993). A problem that might arise in testing CTA memory immediately after training is that the malaise-producing agent, e.g., $\mathrm{LiCl}$, exerts a lingering effect; therefore the negative reinforcer might still be acting while short-term memory is tested. To be able to test short-term CTA memory, we first determined the time window of the behavioral effects of intraperitoneal $\mathrm{LiCl}$ injection under the conditions used by us in CTA training. The most prominent behavioral index of malaise in CTA training is LOB (see Materials and Methods) (Meachum and Bernstein, 1990). LOB was observed during a time window of $<2 \mathrm{hr}$ after the injection of $\mathrm{LiCl}$ intraperitoneally (Fig. 3). Rearing (Parker, 


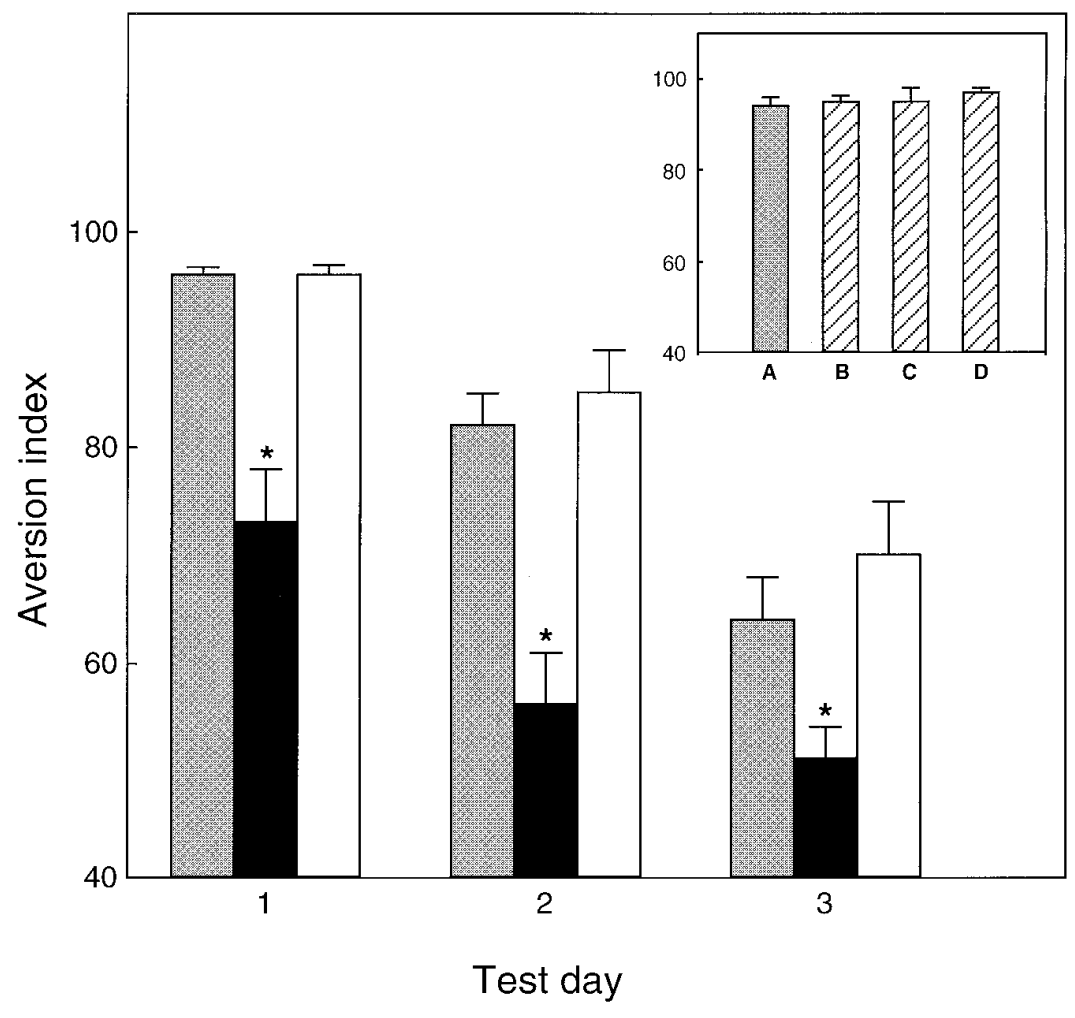

Figure 2. Impairment of CTA by ODNs antisense to CREB in the amygdala. Aversion indices are plotted versus the test day. In each test day, the left (shaded) bar depicts the aversion index of normal CTA control animals $(n=23)$; the center (solid) bar depicts the aversion index of animals locally microinjected into the amygdala with antisense ODNs $(n=16)$; and the right (open) bar shows the index of animals receiving sense ODNs $(n=11)$. All microinjections into the amygdala were performed $14 \mathrm{hr}$ before CTA training, as detailed in Materials and Methods. Inset, Spatial and temporal specificity of the antisense effect. The shaded bar depicts the result for normal controls; the hatched bars show the results for rats microinjected with antisense. $A$, Normal CTA animals; $B$, antisense microinjected $14 \mathrm{hr}$ before training $2 \mathrm{~mm}$ above the stereotaxic coordinates used for injection into the amygdala; $C$, antisense microinjected into the amygdala $14 \mathrm{hr}$ before the first memory test, i.e., $36 \mathrm{hr}$ after the completion of training; $D$, antisense microinjected into the amygdala $7 \mathrm{~d}$ before CTA training; $n=6$ animals in each group. Asterisks indicate significance for pair comparison in which a Scheffe contrast test was used with an $\alpha$ of 0.05 .
1982), which was taken as an indicator of residual unrest, was observed for a longer period (Fig. 3). We concluded that the malaise induced by the negative reinforcer dissipated within $<2$ $\mathrm{hr}$, and that behavior completely returned to normal within another 2-3 hr. We then proceeded to test the effect of CREB antisense in the amygdala on short-term CTA memory. The antisense was microinjected into the amygdala as above, $14 \mathrm{hr}$ before the beginning of CTA training, and the animals were tested at 2 or $4 \mathrm{hr}$ (short-term memory) and again at $72 \mathrm{hr}$ (long-term memory) after the completion of training. As can be seen in Figure 3, CREB antisense had a significant effect only on long-term memory $(p<0.001)$. The data of $4 \mathrm{hr}$, a point at which LOB is long undetected, and even rearing is negligible, clearly demonstrate that the aversion observed was not attributable to the lingering effects of the UCS but, rather, was attributable to memory. There was no difference in the amount of liquid consumed by the rats at 2 and $4 \mathrm{hr}(5.6 \pm 0.6$ and $5.5 \pm 0.2 \mathrm{ml}$, respectively), and combined with the liquid consumed in training $(5 \mathrm{ml})$, it was only slightly below the total amount drank at $72 \mathrm{hr}$ $(11.5 \pm 0.3 \mathrm{ml})$. Interestingly, no extinction was seen when rats tested for short-term memory were retested for long-term memory, whereas such extinction is usually observed in repetitive long-term memory tests (e.g., Fig. 2).

\section{CREB antisense had no effect on retrieval}

We microinjected CREB antisense ODNs into the amygdala 36 hr after training, i.e., $14 \mathrm{hr}$ before the first memory test. Under these conditions, no effect on CTA memory was detected (Fig. 2, inset). Hence, CREB antisense in the amygdala had no effect on detection of saccharin, on its conditioned hedonic valence, or on other sensory or motor faculties involved in the acquired rejection of the saccharin solution.

\section{DISCUSSION}

Although ample data indicate that the amygdala subserves CTA (for review, see Yamamoto et al., 1994), its role is still debated, and questions remain regarding the timing of its contribution (i.e., acquisition, retention, or retrieval), its role in the circuits (either modulatory and transient or long-lasting), and the identity of the subnuclei involved. Most of the data are based on lesions and suggest that the effect on CTA depends on the type of lesion (transient or permanent, affecting fibers of passage or sparing them), the timing of the lesion relative to training and testing, and the location of the damage. Whereas some studies unveiled no effect of amygdala lesions on CTA (Kemble et al., 1979; Hatfield et al., 1992; Galaverna et al., 1993), others showed that such lesions, especially of the basolateral amygdala (BLA) (Fitzgerald and Burton, 1983; Simbayi et al., 1986; Yamamoto and Fujimoto, 1991) and also the CeA (Lasiter and Glanzman, 1982, 1985; Roldan and Bures, 1994; Schafe and Bernstein, 1996), disrupted CTA. Transient inhibition of protein synthesis in the central amygdala throughout training also disrupted CTA (Lamprecht and Dudai, 1996). Not all of the data are based on inference of function from dysfunction; some correlative data are also available. The latter include single-unit recording from the BLA and CeA (Yasoshima et al., 1995) and in situ hybridization analysis that revealed increased expression of the IEGs mRNA in the $\mathrm{CeA}$ after administration of $\mathrm{LiCl}$ intraperitoneally, the UCS conventionally used in CTA (Lamprecht and Dudai, 1995).

All in all, the majority of available information does implicate the amygdala, including $\mathrm{CeA}$, in some aspects of CTA. To determine further whether $\mathrm{CeA}$ is obligatory for CTA and to elucidate the molecular mechanisms involved, we decided to inhibit the expression of IEGs transiently in the amygdala in CTA training and testing. We recently reported that microinjec- 
Figure 3. CREB antisense in the amygdala selectively impairs long- but not short-term memory. Aversion indices are plotted versus the time of test after CTA training. Closed circles, Rats microinjected with CREB antisense ODNs 14 hr before CTA training; open circles, control animals injected with saline instead of CREB antisense. The figure also depicts the time windows of LOB behavior (dark gray) and rearing (light gray), i.e., measures of the on-line effect of the malaise inducing-agent as a function of the time after $\mathrm{LiCl}$ injection intraperitoneally. For further details see Results. The asterisk indicates significance for pair comparison in which a Scheffe contrast test was used with an $\alpha$ of 0.05 .

tion of ODNs antisense to c-fos into the central amygdala before CTA training impaired CTA memory tested a few days later (Lamprecht and Dudai, 1996). c-fos is a CRE-regulated IEG. We now argue that CREB in the amygdala is indeed required for normal CTA memory, but only for the long-term form of it; short-term CTA memory, as well as retrieval of memory once it has been formed, is unaffected. Although the sphere of drug diffusion in the brain exceeds the $\mathrm{CeA}$, the only salient common feature of all of the sections analyzed by us after microinjection of either india ink or FITC-CREB was the bilateral involvement of substantial portions of the CeA (see Materials and Methods). Furthermore, although part of the microinjected solution diff used dorsolaterally along the cannula track, microinjection $2 \mathrm{~mm}$ above the CeA had no effect on CTA (see Results). We therefore consider the $\mathrm{CeA}$ a prime candidate for the site of action of the antisense in this study.

Before proceeding to discuss potential implications of our results on the understanding of CTA mechanisms, a few remarks on antisense technology are pertinent. Whereas ODNs are rapidly degraded in vivo, phosphorothioate-modified ODNs display markedly increased stability (Campbell et al., 1990). However, the chemical modification may also increase toxicity (Chiasson et al., 1994). In addition to inhibiting the interaction of the target mRNA with the ribosome and translation factors, phosphorothioate ODNs form an ODNs-mRNA hybrid that is a substrate for RNase $\mathrm{H}$, which recognizes DNA-RNA hybrids and cleaves the RNA (Gao et al., 1991). Other effects include inhibition rather than activation of RNase $\mathrm{H}$ and inhibition of DNA polymerases (Gao et al., 1991; Helene, 1991), and interaction with other proteins (Perez et al., 1994). Some of these effects are nonsequence-specific and increase with the length of the ODNs, the dose, and the duration of application (Gao et al., 1991; Helene, 1991; Chiasson et al., 1994). In that respect, the ability to use a

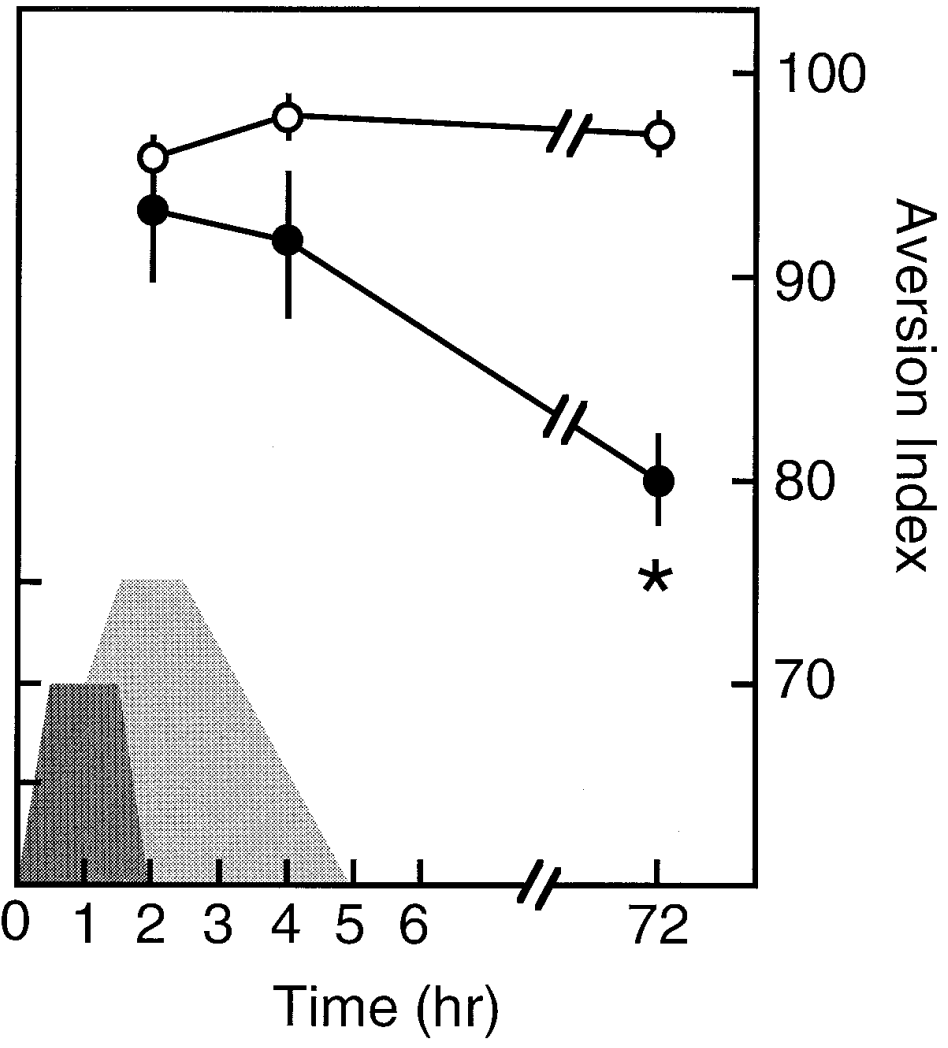

single local ODNs application, because of the single-trial learning situation in the CTA paradigm, is an advantage. We selected injection of the ODNs into the amygdala once, $14 \mathrm{hr}$ before the beginning of training, because this timing was previously shown to be effective in inhibition of CREB translation by CREB antisense in the rat brain in vivo (Konradi et al., 1994). The reduction of expression that we obtained was partial, similar to that previously reported in the nucleus accumbens (Widnell et al., 1996). Apparently, the dependence of brain function on the level of constitutively expressed CREB is rather critical and sensitive to a decrease of even $\sim 40 \%$.

Several observations support the assumption that the effects were specific to the CREB antisense ODNs: (1) there was a clear differential effect of the sense and antisense ODNs on CTA behavior and on expression of CREB protein in the injected amygdaloid region; (2) CREB antisense did not alter the level of ATF-2 and $\mathrm{PKC}_{\gamma}$; (3) the effect on expression of the CREB protein was transient, with a time course compatible with the published life span of the ODNs in vivo; (4) the antisense caused no differential anatomical damage (although it should be noted that the resolution of our anatomical analysis was limited) (see Coggeshell, 1992); (5) the treatment had no effect on retraining; (6) the antisense did not affect retrieval under conditions that affected conditioning; and (7) the effect was confined to long- but not short-term memory. Taken together, the aforementioned observations demonstrate that under the conditions used in this study, the antisense had no short-term toxicity relevant to the expression of CTA behavior and no long-term toxic effects either. We cannot rule out the possibility that in addition to its specific suppression of CREB expression, CREB antisense modulates another cellular mechanism, which is unaffected by CREB sense; this type of reservation applies generally to studies using inhibitors to infer function from dysfunction. 
The amygdala has been repeatedly implicated in learning, especially aversive and emotional learning (McGaugh et al., 1993; Davis et al., 1994; Gallagher and Holland, 1994; Yamamoto et al., 1994; Maren and Fanselow, 1996; Rogan and LeDoux, 1996). In general, two major roles for amygdala involvement in learning and memory were proposed (without being mutually exclusive). One is that the amygdala plays a temporary role in modulating other brain regions in the process of acquisition and consolidation of aversive memories (McGaugh et al., 1993). The other is that experience-dependent changes take place in amygdaloid circuits, which subserve long-term storage of the modified internal representation of the aversive response. The latter hypothesis underlies circuit models of fear conditioning (Davis et al., 1994; Maren and Fanselow, 1996; Rogan and LeDoux, 1996). These models distinguish in the amygdala two subsystems that subserve fear conditioning: the basolateral complex, in which sensory information converges from cortical and subcortical areas, possibly to form the CS-UCS association in fear conditioning; and the CeA, which receives projections from the basolateral complex and projects to other brain regions involved in the fear response. The $\mathrm{CeA}$ also receives direct projections from the parabrachial nucleus and the insular cortex, which might be particularly significant in CTA.

Our data favor two notions: (1) the role of the central amygdala in CTA is not limited to fibers of passage, as might be inferred from some excitotoxic lesion data (Dunn and Everitt, 1988); and (2) the role of the central amygdala in CTA is not confined to transient modulation of other circuits during training, and involves lasting modifications in circuits that include amygdala neurons. The functional nature of such proposed modifications is not addressed in the present study. They may bear on specific CTA representations or more general representations of emotion and fear, and may last for only days or much longer. A role of amygdala in storage of experience-dependent representations is in consonance with the types of models suggested for fear conditioning (Davis et al., 1994; Maren and Fanselow, 1996; Rogan and LeDoux, 1996). Furthermore, it is tempting to speculate that some amygdala circuit modules that subserve fear conditioning are also shared with CTA.

The molecular mechanisms implicated in the aforementioned postulated modifications of amygdala in CTA involve CREB. This is similar to the picture obtained in a number of other experimental systems, which implicate regulation of CREB and CRE-mediated gene expression, via cAMP- and $\mathrm{Ca}^{2+}$-regulated signal transduction cascades, in long-term circuit alterations subserving neural development or consolidation of long-term memory (Bourtchuladze et al., 1994; Bartsch et al., 1995; Yin et al., 1995; Impey et al., 1996; Liu and Graybiel, 1996; Guzowski and McGaugh, 1997). An increase in c-Fos, a CRE-regulated IEG product, has been reported to take place in the nucleus of the solitary tract (NTS) in CTA-trained rats in response to the CS (Swank and Bernstein, 1994; Houpt et al., 1995). The latter observation raises the possibility that either in the NTS c-Fos elevation is correlated with, but not obligatory for, CTA expression, or that whenever a trained individual is reexposed to the CS in testing, relearning takes place, which involves IEG modulation in the NTS. Interestingly, this IEG modulation was not detected in the NTS ipsilateral to a unilaterally lesioned amygdala (Schafe and Bernstein, 1996). Infusion of $c$-fos antisense into the fourth ventricle reduced c-Fos-positive nuclei in the NTS and impaired acquisition and extinction of CTA in mice (Swank et al., 1996), but such infusion lacks the site specificity required to make a firm conclusion about the obligatory role of c-fos in NTS in CTA.

If we now return to the questions posed at the beginning of this discussion, concerning the role and timing of amygdala contribution to CTA, the following answers may be offered: the amygdala is required for proper encoding of long-term CTA memory; CeA is involved; and it undergoes cellular alterations that suggest some long-term change. We also propose that whatever circuit mechanisms endow taste aversion conditioning with its unique tolerance to a very long CS-UCS interval, they are based on molecular mechanisms that also subserve many other types of learning. Our data further reinforce the notion that CREB is a component of such a cross-task, cross-species, and cross-phyla molecular universal.

\section{REFERENCES}

Bartsch D, Ghirardi M, Skehel PA, Karl KA, Herder SP, Chen M, Bailey CH, Kandel ER (1995) Aplysia CREB2 represses long-term facilitation: relief of repression converts transient facilitation into long-term functional and structural change. Cell 83:979-992.

Bermudez-Rattoni F, Grijalva CV, Kiefer SW, Garcia J (1986) Flavorillness aversions: the role of the amygdala in the acquisition of tastepotentiated odor aversions. Physiol Behav 38:503-508.

Bourtchuladze R, Frenguelli B, Blendy J, Cioffi D, Schutz G, Silva AJ (1994) Deficient long-term memory in mice with a targeted mutation of the cAMP-responsive element-binding protein. Cell 79:59-68.

Bures J, Buresova O, Kriveanek J (1988) Brain and behavior: paradigms for research on neuronal mechanisms. New York: Wiley.

Burnette WW (1981) Western blotting: electrophoretic transfer of proteins from sodium dodecyl sulfate-polyacrylamide gels to unmodified nitrocellulose and radiographic detection with antibody and radioiodinated protein A. Anal Biochem 112:195-203.

Campbell JM, Bacon TA, Wickstrom E (1990) Oligodeoxynucleoside phosphorothioate stability in subcellular extracts, culture media, sera and cerebrospinal fluid. J Biochem Biophys Methods 20:259-267.

Carew TJ (1996) Molecular enhancement of memory formation. Neuron 16:5-8.

Chiasson BJ, Armstrong JN, Hooper ML, Murphy PR, Robertson HA (1994) The application of antisense oligonucleotide technology to the brain: some pitfalls. Cell Mol Neurobiol 14:507-520.

Cirelli C, Pompeiano M, Tononi G (1995) In vivo antisense approaches to the role of immediate early gene expression in the brain. Regul Pept 59:151-162.

Coggeshell RE (1992) A consideration of neural counting methods. Trends Neurosci 15:9-13.

Davis M (1992) The role of the amygdala in fear and anxiety. Annu Rev Neurosci 15:353-375.

Davis M, Rainnie D, Cassell M (1994) Neurotransmission in the rat amygdala related to fear and anxiety. Trends Neurosci 17:208-241.

Domjan M (1980) Ingestional aversion learning: unique and general processes. Adv Study Behav 11:275-336.

Dunn LT, Everitt BJ (1988) Double dissociations of the effects of amygdala and insular cortex lesions on conditioned taste aversion, passive avoidance, and neophobia in the rat using the excitatoxin ibotonic acid. Behav Neurosci 102:3-23.

Dustin P (1984) Microtubules. Ed 2. Berlin: Springer.

Fitzgerald RE, Burton MJ (1983) Neophobia and conditioned taste aversion deficits in the rat produced by undercutting temporal cortex. Physiol Behav 30:203-206.

Frank DA, Greenberg ME (1994) CREB: a mediator of long-term memory from mollusk to mammals. Cell 79:5-8.

Galaverna OG, Seeley RJ, Berridge KC, Grill HJ, Epstein AN, Schulkin J (1993) Lesions of the central nucleus of the amygdala. I. Effects of taste reactivity, taste aversion learning and sodium appetite. Behav Brain Res 59:11-17.

Gallagher M, Holland PC (1994) The amygdala complex: multiple roles in associative learning and attention. Proc Natl Acad Sci USA 91:11771-11776.

Gallo M, Roldan G, Bures J (1992) Differential involvement of gustatory insular cortex and amygdala in the acquisition and retrieval of conditioned taste aversion in rats. Behav Brain Res 52:91-97.

Gao W-Y, Han F-S, Storm C, Egan W, Cheng Y-C (1991) Phosphoro- 
thioate oligonucleotides are inhibitors of human DNA polymerases and RNase H: implications for antisense technology. Mol Phramacol 41:223-229.

Garcia J (1981) Tilting at paper mills of academe. Am Psychol 36:149-158.

Garcia J, Kimmeldorf DJ, Koelling RA (1955) Conditioned aversion to saccharin resulting from exposure to gamma radiation. Science 122:157-158.

Ginty DD, Kornhuaser JM, Thompson MA, Bading H, Mayo KE, Takahashi JS, Greenberg ME (1993) Regulation of CREB phosphorylation in the suprachiasmatic nucleus by light and a circadian clock. Science 260:238-241.

Guzowski JF, McGaugh JL (1997) Antisense oligodeoxynucleotidemediated disruption of hippocampal cAMP response element binding protein levels impairs consolidation of memory for water maze training. Proc Natl Acad Sci USA 94:2693-2698.

Hatfield T, Graham PW, Gallagher M (1992) Taste-potentiated odor aversion learning: role of the amygdaloid basolateral complex and central nucleus. Behav Neurosci 106:286-293.

Hebb DO (1949) The organization of behavior: a neuropsychological theory. New York: Wiley.

Helene C (1991) Rational design of sequence-specific oncogene inhibitors based on antisense and antigene oligonucleotides. Eur $\mathrm{J}$ Cancer 11:1466-1471.

Houpt TA, Berlin RA, Smith GP (1995) Altered induction of c-Fos in the central nucleus of the amygdala $(\mathrm{CeN})$ correlated with conditioned taste aversion expression. Soc Neurosci Abstr 21:660.5.

Impey S, Mark M, Villacres EC, Poser S, Chavkin C, Storm DR (1996) Induction of CRE-mediated gene expression by stimuli that generate long-lasting LTP in area CA1 of the hippocampus. Neuron 16:973-982.

Kam Z, Chen H, Sedat JW, Agard DA (1992) Analysis of threedimensional image data: display and feature tracking. In: Electron tomography. Three-dimensional imaging with the transmission electron microscope (Frank J, ed), pp 237-256. New York: Plenum.

Kam Z, Jones MO, Chen H, Agard DA, Sedat JW (1993) Design and construction of an optimal illumination system for quantitative widefield multi-dimensional microscopy. Bioimaging 1:71-81.

Kemble ED, Studelska DR, Schmidt MK (1979) Effects of central amygdaloid nucleus lesions on the ingestion, taste reactivity, exploration and taste aversion. Physiol Behav 22:789-793.

Konradi C, Rebecca LC, Heckers S, Hyman SE (1994) Amphetamine regulates gene expression in rat striatum via transcription factor CREB. J Neurosci 14:5623-5634.

Laemmli UK (1970) Cleavage of structural proteins during the assembly of the head of bacteriophage $\mathrm{T}_{4}$. Nature 227:680-685.

Lamprecht R, Dudai Y (1995) Differential modulation of brain immediate early genes by intraperitoneal LiCl. NeuroReport 7:289-293.

Lamprecht R, Dudai Y (1996) Transient expression of c-Fos in rat amygdala during training is required for encoding conditioned taste aversion memory. Learn Mem 3:31-41.

Lasiter PS, Glanzman DL (1982) Cortical substrates of taste aversion learning: dorsal prepiriform (insular) lesions disrupt taste aversion learning. J Comp Physiol Psychol 96:376-392.

Lasiter PS, Glanzman DL (1985) Cortical substrates of taste aversion learning: involvement of dorsolateral amygdaloid nuclei and temporal neocortex in taste aversion learning. Behav Neurosci 99:257-276.
LeDoux JE (1993) Emotional memory: in search of systems and synapses. Ann NY Acad Sci 702:149-157.

Liu F-C, Graybiel AM (1996) Saptiotemporal dynamics of CREB phosphorylation: transient vs. sustained phosphorylation in the developing striatum. Neuron 17:1-20.

Maren S, Fanselow MS (1996) The amygdala and fear conditioning: has the nut been cracked? Neuron 16:237-240.

McGaugh JL, Introini-Collison IB, Cahill LF, Castellano C, Dalmaz C, Parent MB, Williams CL (1993) Neuromodulatory systems and memory storage: role of amygdala. Behav Brain Res 58:81-90.

Meachum CL, Bernstein IL (1990) Conditioned responses to a taste conditioned stimulus paired with lithium chloride administration. Behav Neurosci 104:711-715

Parker LA (1982) Nonconsummatory and consummatory behavioral CRs elicited by lithium- and amphetamine-paired flavors. Learn Motiv 13:281-303.

Paxinos G, Watson C (1986) The rat brain in stereotaxic coordinates, Ed 2. San Diego: Academic.

Perez JR, Li Y, Stein CA, Majumder S, van Oorschot A, Narayanan R (1994) Sequence-independent induction of SP1 transcription factor activity by phosphorothioate oligonucleotides. Proc Natl Acad Sci USA 91:5959-5961.

Rogan MT, LeDoux JE (1996) Emotion: systems, cells, synaptic plasticity. Cell 469-475.

Roldan G, Bures J (1994) Tetrodotoxin blockade of amygdala overlapping with poisoning impairs acquisition of conditioned taste aversion in rats. Behav Brain Res 65:213-219.

Rosenblum K, Meiri N, Dudai Y (1993) Taste memory: the role of protein synthesis in gustatory cortex. Behav Neural Biol 59:49-56.

Schafe GE, Bernstein IL (1996) Forebrain contribution to the induction of a brainstem correlate of conditioned taste aversion: I. The amygdala. Brain Res 741:109-116.

Simbayi LC, Boakes RA, Burton MJ (1986) Effects of basolateral amygdala lesions on taste aversions produced by lactose and lithium chloride in the rat. Behav Neurosci 100:455-465.

Swank MW, Bernstein IL (1994) c-Fos induction in response to a conditioned stimulus after single trial taste aversion learning. Brain Res 636:202-208.

Swank MW, Ellis AE, Cochran BN (1996) c-Fos antisense blocks acquisition and extinction of conditioned taste aversion memory. NeuroReport 7:1866-1870.

Widnell KL, Self DW, Lane SB, Russell DS, Vaidya VA, Miserendino MJD, Rubin CS, Duman RS, Nestler EJ (1996) Regulation of CREB expression: in vivo evidence for a functional role in morphine action in the nucleus accumbens. J Pharmacol Exp Ther 276:306-315.

Yamamoto T, Fujimoto Y (1991) Brain mechanisms of taste aversion learning in the rat. Brain Res Bull 27:403-406.

Yamamoto T, Shimura T, Sako N, Yasoshima Y, Sakai N (1994) Neural substrates for conditioned taste aversion in the rat. Behav Brain Res 65:123-137.

Yasoshima Y, Shimura T, Yamamoto T (1995) Single unit responses of the amygdala after conditioned taste aversion in conscious rats. NeuroReport 6:2424-2428.

Yin JCP, Del Vecchio M, Zhou H, Tully T (1995) CREB as a memory modulator: induced expression of a dCREB2 activator isoform enhances long-term memory in Drosophila. Cell 81:107-115. 